\title{
Light scattering study of microemulsions and its relation to percolation phenomena
}

\author{
A. M. Cazabat, D. Chatenay (*), D. Langevin and A. Pouchelon (**) \\ Laboratoire de Spectroscopie Hertzienne de l'E.N.S., 24, rue Lhomond, 75231 Paris Cedex 05, France
}

(Reçu le 3 juin 1980, accepté le 21 juillet 1980)

\begin{abstract}
Résumé. - Les caractéristiques de la lumière diffusée par des microémulsions au voisinage d un seuil de percolation indiquent l'existence de forces attractives à longue portée entre micelles. Les interactions hydrodynamiques sont également présentes, mais n'ont pas de comportement à longue portée.
\end{abstract}

\begin{abstract}
The characteristics of the light scattered by microemulsions in the vicinity of a percolation threshold indicate the existence of strong and long range attractive forces between micelles. Hydrodynamic interactions are also present but show no evidence of long range character.
\end{abstract}

1. Introduction. - Water in oil microemulsions are transparent dispersions of water droplets surrounded by amphiphilic molecules in a continuous organic medium. Light scattering techniques already allowed investigators to obtain much information about droplet sizes and interaction forces in these media [1-6]. The characteristics of the scattered light are usually typical of brownian motion of small particles interacting through short range potentials (short compared to optical wavelengths) : the correlation function of the scattered electric field is exponential and both the scattered intensity and the diffusion coefficient (deduced from the correlation time) are independent of the scattering angle.

In some cases however : (vicinity of a gel [7], of a flocculation point for microemulsions made with polymeric materials [4]), the correlation function of the scattered electric field becomes non exponential. The interpretation of these features is not yet clear. In the last reference, the authors remark that they are accompanied by interparticle packing : electron micrographs show the presence of compact networklike clusters made of individual droplets [8].

Our experiments deal with microemulsions made with an anionic soap, far from a gelation point. In a previous paper [6], we mentioned that when attractive forces between droplets are strong, at volume fractions close to 0.1 , the diffusion coefficient depends on scattering angle. Such volume fractions correspond

(*) Recipient of a fellowship from the Institut Français du Pétrole. (**) Permanent address : Rhône Poulenc, Centre de Recherches de Decines, 69150 Decines, France. to the percolation threshold already observed in electrical conductivity measurements by Lagües et al. [9]. In the stirred percolation model proposed by Lagües to interpret these measurements, it is assumed that the droplets behave as randomly distributed hard spheres. Transient clusters are statistically formed. Above a critical volume fraction $\varphi_{c}$, an infinite path appears through the droplets. The electrical conductivity increases steeply (several orders of magnitude) around $\varphi_{\mathrm{c}}$.

In the present paper, we describe a preliminary study of the characteristics of the scattered light in the vicinity of the percolation threshold for microemulsions in which attractive forces are strong.

A careful analysis of the autocorrelation function showed no evidence of departure from exponential behaviour. However, the diffusion coefficient was found to depend on scattering angle. This is accompanied by an angular dependence of the scattered intensity which has also been measured.

Similar features were already found in other systems when the correlation length becomes of the order of the optical wavelength : fluid near a critical point [10], charged macromolecules solutions [11], latex particles dispersions $[12,13]$. In the two last cases the autocorrelation function has a pronounced non exponential behaviour. Pusey [12] attributed this effect to the existence of two slow processes in the medium : diffusion of individual particles across solvent molecules and changes of the instantaneous configuration of particle neighbours. The mean interparticle spacing being large, the characteristic time $\tau_{I}$ of the second process is long $(\sim 200 \mu \mathrm{s})$ : the correlation function 
decay is determined by free particle motion for $\tau<\tau_{\mathrm{I}}$ and by interparticle forces for $\tau>\tau_{\mathrm{I}}$. In our present problem, the mean interparticle spacing is not significantly different from particle radius :

$$
d \sim R\left(4 \varphi^{-1}\right)^{1 / 3} \sim 3.5 R \text { for } \varphi \sim 0.1 ;
$$

$\tau_{\mathrm{I}}$ is very small ( $\left.\sim 10 \mathrm{~ns}\right)$ compared to correlation delay times. Consequently, the particle concentration can be considered as the only slowly decaying property, and its correlation function will be exponential as observed.

The analogy between the present problem and critical phenomena is closer. In both cases, the correlation function for the concentration is exponential (no coupling with others hydrodynamic variables).

Near a critical point, a picture of a fluid can also been made with transient clusters of size $\xi$. The correlation length $\xi$ is the range over which the concentration fluctuations are correlated and diverges at the critical point. In the percolation problem, the correlation length is the spatial size of the largest clusters and diverges also beyond the percolation threshold. However, the analogy cannot be driven too far : indeed the scattering of light by a percolating system does not usually diverge near the threshold : the important fluctuations are not in the concentration but in the connectivity $[14,15]$.

An explanation of the variation of both scattered intensity and diffusion coefficient with scattering angle in our experiments will be given with the help of the recent theories of brownian motion of interacting particles $[16,17]$.

2. Samples and experimental set up. - The samples are quaternary mixtures of water, sodium dodecylsulfate, toluol as oil, butanol 1 (microemulsion ATB) or pentanol 1 (microemulsion ATP) as cosurfactant. The volume fraction $\varphi$ of the droplets has been calculated according to the procedure described in reference [5]. The electrical conductivity has been measured for the two kind of samples (using a Metrohm E518

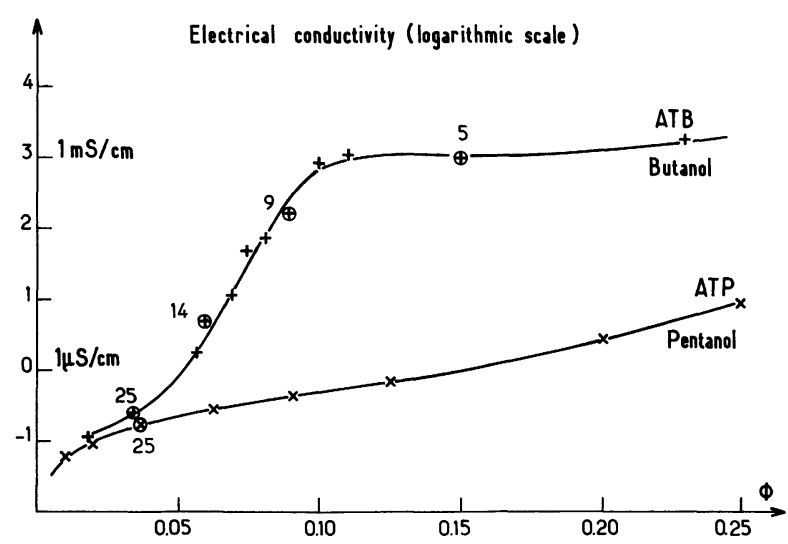

Fig. 1. - Semi logarithmic plot of electrical conductivity variation versus droplets volume fraction for the two different types of microemulsions : toluene-butanol and toluene-pentanol. The circles and numbers are relative to the samples that were studied in more detail in the light scattering experiments. conductimeter) as a function of concentration. The results are reported on figure 1 . For microemulsion ATP the variation of electrical conductivity with volume fraction remains slow. But microemulsion ATB shows evidence of percolation phenomenon around $\varphi_{\mathrm{c}} \sim 0.08$.

The light source is an argon ion laser CR3 giving an output power of $0.5 \mathrm{~W}$ at a wavelength $\lambda=4880 \AA$. Close to percolation threshold, this power has been considerably reduced in order to avoid heating effects in the samples.

Intensity measurements were performed in $1 \mathrm{~mm}$ optical path length cells (Hellma) immersed in a toluol bath that exactly matches the indices (microemulsion, toluol and glass have practically the same index : 1.485 to 1.5$)$. The illuminated volume was kept constant and entirely imaged in the photocathode of the photomultiplier in order to avoid angular corrections. For $\theta<15^{\circ}$ or $\theta>165^{\circ}$ and microemulsions far from percolation threshold the amount of light scattered elastically becomes significant $(5 \%$ for microemulsion 5 ATB at $\theta=15^{\circ}$ ).

The correlation function of the scattered light was also recorded using a full parallel correlator, with 100 channels [5]. The detection is homodyne, excepted at the lowest scattering angles $\theta\left(\theta<5^{\circ}\right)$. The light scattered by the cell walls plays then the role of local oscillator, and the detection becomes heterodyne.

The scattering angles $\theta$ were varied from $0.65^{\circ}$ to $140^{\circ}$. The scattering wave vector $q=\frac{4 \pi n}{\lambda} \sin \frac{\theta}{2}$ ( $n$ is the microemulsion index) varies then from

$$
q=2.2 \times 10^{3} \mathrm{~cm}^{-1} \text { to } q=3.6 \times 10^{5} \mathrm{~cm}^{-1} \text {. }
$$

This allows to explore a wide range of length scales ( $q^{-1}$ between $5 \mu \mathrm{m}$ and $300 \AA$ ). In all cases multiple scattering was found negligible $\left(\lesssim 10^{-2}\right)$.

3. Experimental results. - 3.1 Autocorrelation FUNCTION $g_{2}(\tau)$. - The autocorrelation function $g_{2}(\tau)$ has been fitted with the simple form :

$$
g_{2}(\tau)=1+g_{1}^{2}(\tau)=1+\mathrm{e}^{-2 D q^{2} \tau} .
$$

The diffusion coefficient $D$ is independent of $q$ far from percolation threshold for microemulsions ATB and in all cases for microemulsions ATP. This is characteristic of Brownian motion of small particles and short range interactions [18]. When the percolation threshold is approached in microemulsions ATB, $D$ becomes dependent on $q$.

The variation of diffusion coefficient with volume fraction for $\theta=90^{\circ}$ is represented on figure 2. Its extrapolation $D_{0}$ to $\varphi=0$ allows the determination of the hydrodynamic radius [18] :

$D_{0}=k T / 6 \pi \eta R_{0} \quad \eta$ viscosity of the continuous phase. $R_{0}=41 \AA$ for microemulsions ATB (toluol-butanol) $R_{0}=45 \AA$ for microemulsions ATP (toluol-pentanol) 


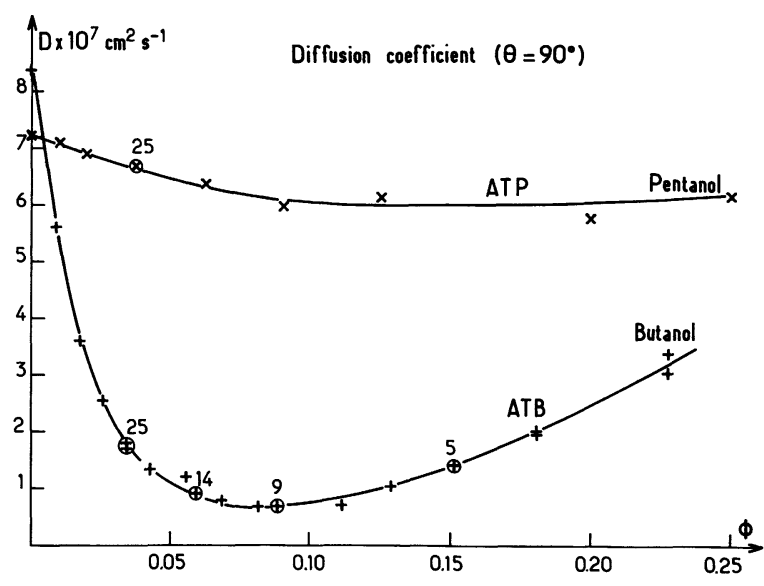

Fig. 2. - Diffusion coefficient deduced from the autocorrelation function : $g_{2}(\tau)=\exp \left(-2 D q^{2} \tau\right)+1$ at a scattering angle $\theta=90^{\circ}$, versus volume fraction. The extrapolation to $\varphi=0$ gives $D_{0}$.

The negative slope of $D(\varphi)$ at the origin indicates that interactions are attractive, and much stronger in the case of microemulsions ATB (large negative slope) than for microemulsions ATP [6].

The variation of diffusion coefficient with $\theta$ is represented on figure 3 for $\varphi$ values close to percolation threshold. The exponential character of $g_{2}(\tau)$

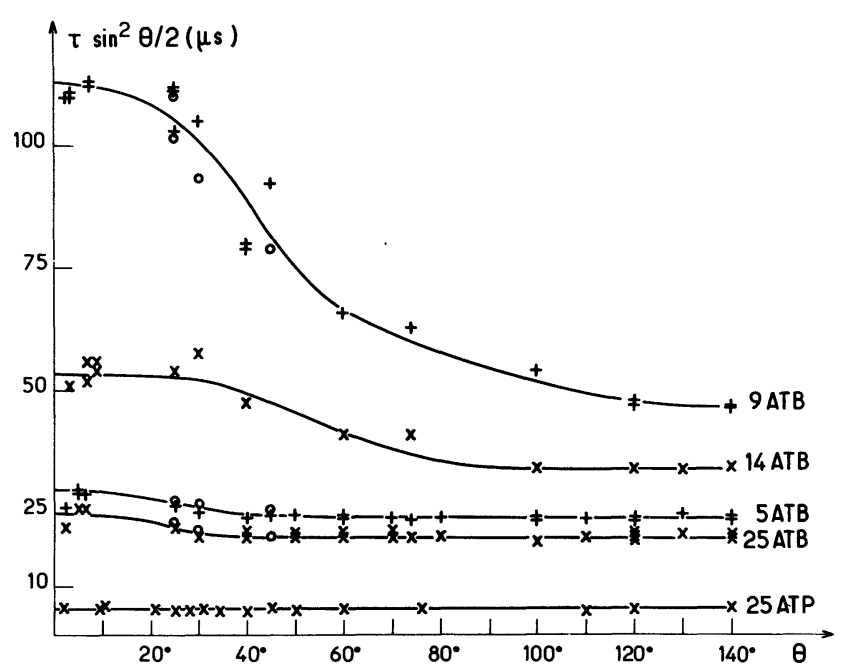

Fig. 3. - Correlation times $\tau=\left(2 D q^{2}\right)^{-1}$ multiplied by $\sin ^{2} \theta / 2$ versus scattering angle $\theta ; \tau \sin ^{2} \theta / 2$ is a quantity inversely proportional to the diffusion coefficient $D$. The droplets volume fractions for the different microemulsions can be found in figures 1 and 2 .

has been analysed with special care. For this purpose, a fit using the method of cumulants has been done :

$$
\ln \left(g_{2}(\tau)-1\right)=-\alpha_{1} \tau+\frac{\alpha_{2}}{2} \tau^{2}+0\left(\tau^{3}\right)
$$

$\alpha_{1}=2 D q^{2}$. The variance $v=\sqrt{\alpha_{2}} / \alpha_{1}$ has been found equal to zero in all cases. The quality of the fit is extremely good the mean square deviation between the experimental curve and the exponential

$$
\left(\frac{I}{N} \sum_{i=1}^{N}\left[g_{2}^{\exp }\left(\tau_{i}\right)-g_{2}^{\mathrm{th}}\left(\tau_{i}\right)\right]^{2}\right)^{1 / 2}
$$

is typically of the order of $10^{-3}(N=$ number of channels).

3.2 SCATTERED INTENSITY 1 . - As one approaches the percolation threshold, microemulsions ATB show large intensity angular variations represented on figure 4. The scattered intensity is much smaller for microemulsions ATP, but is independent of $\theta$.

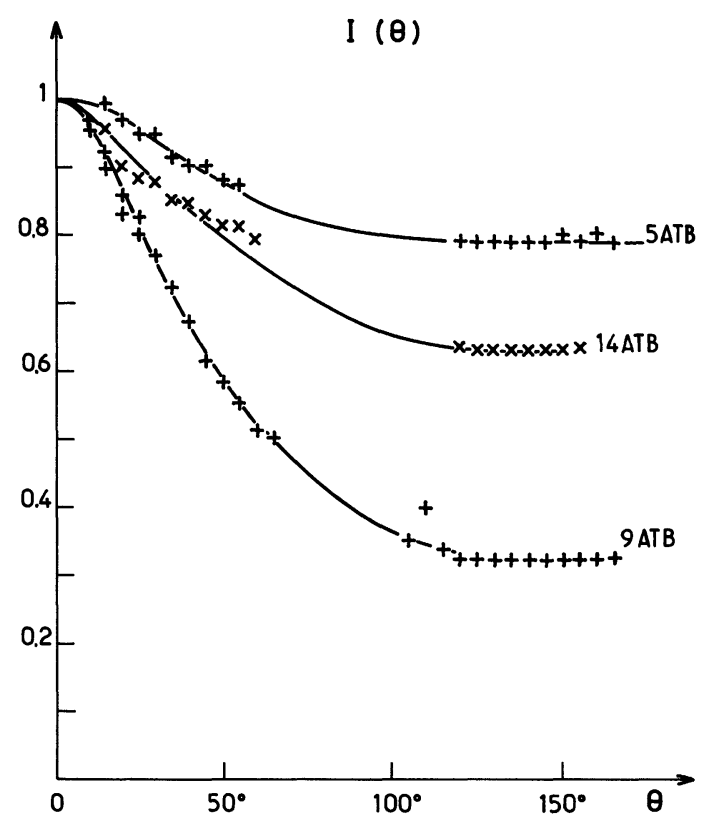

Fig. 4. - Normalized scattered intensity versus scattering angle for different microemulsions ATB. The corresponding volume fraction values can be found figures 1 and 2 .

4. Interpretation. - Light scattering arises mainly from concentration fluctuations of the particles in the solution. Then the scattered intensity is given by [19]':

$$
I(q)=A \varphi I_{0} P(q) S(q)
$$

$I_{0}$ is the intensity of the incident beam, $A$ a constant. $P(q)$ is the particle form factor : $P(q) \approx 1$ for particles of radius $R \sim 50 \AA . S(q)$ is the structure factor :

$$
S(q)=1+\frac{3 \varphi}{R^{3}} \int_{0}^{\infty} r^{2}(g(r)-1) \frac{\sin q r}{q r} \mathrm{~d} r
$$

$g(r)$ being the radial distribution function.

The correlation function of the scattered electric field is proportional to the correlation function of the concentration and follows the memory equation $[11,17]$.

$$
\frac{\mathrm{d} g_{1}}{\mathrm{~d} t}(\tau)=-\frac{1}{S(q)} \int_{0}^{\infty} M(\mathbf{q}, t-\tau) g_{1}(\tau) \mathrm{d} t
$$


If the concentration is the only slowlydecaying property the memory function $M$ is local in time and eq. (5) becomes

$$
\frac{\mathrm{d} g_{1}}{\mathrm{~d} t}(\tau)=-D(q) q^{2} g_{1}(\tau)
$$

with

$$
D(q)=D_{0} \frac{H(q)}{S(q)}
$$

$H(q)$ contains the hydrodynamic interactions [17]. $S(q)$ and $H(q)$ become 1 in the low density limit $(\varphi \rightarrow 0)$. For $q=0$ :

$$
S(0)=\frac{k T}{\partial \pi / \partial \varphi} \times \frac{3}{4 \pi R_{0}^{3}} \quad H(0)=\frac{6 \pi \eta R_{0}}{f} .
$$

The values of $S(q)$ as deduced from intensity data are shown on figure $5\left({ }^{1}\right)$. They seem unusually large, but is must be kept in mind that the particle concentration is large and that the effect of interaction forces is important. The values of $H(q)$ have also been derived using eq. (6) and the measured values of $D(q)$ (Fig. 3) and $S(q)$ (Fig. 5). $H(q)$ differs slightly from the low density limit value of 1 due to hydrodynamic interactions $\left({ }^{1}\right)$. But its variation with $q$ falls beyond experimental accuracy.

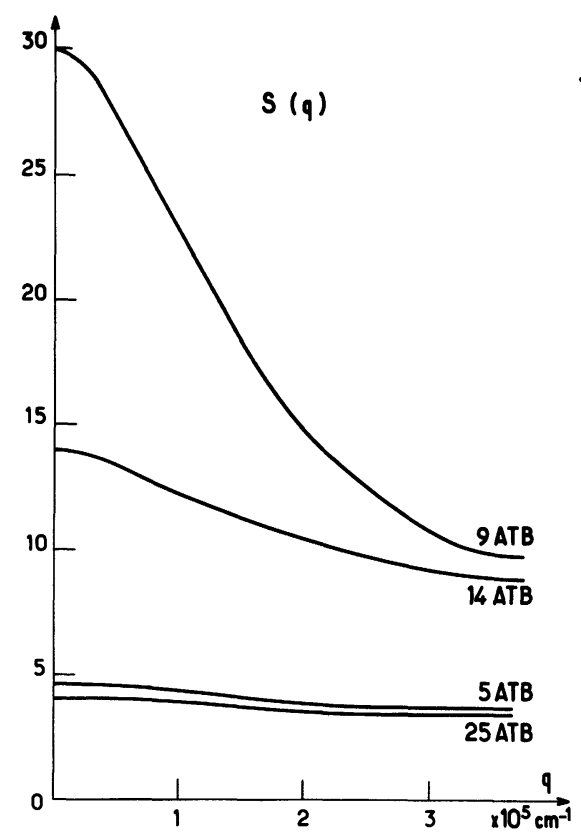

Fig. 5. $-q$ dependence of the structure factor $S$ for different volume fractions in microemulsions ATB (smoothed curves).

5. Conclusion. - A light scattering study of microemulsions in which strong attractive forces are present

${ }^{(1)}$ The variations with $q$ of $S(q)$ and $H(q)$ are known with a good precision since they are deduced directly from $I(\theta)$ and $D(\theta)$ (Figs. 3 and 4). But the absolute values of $S(q)$ and $H(q)$ are much less accurate since they involve the extrapolation of $I / \varphi$ to $\varphi=0$, domain where $I$ is a very rapid function of $\varphi\left[{ }^{6}\right]$.
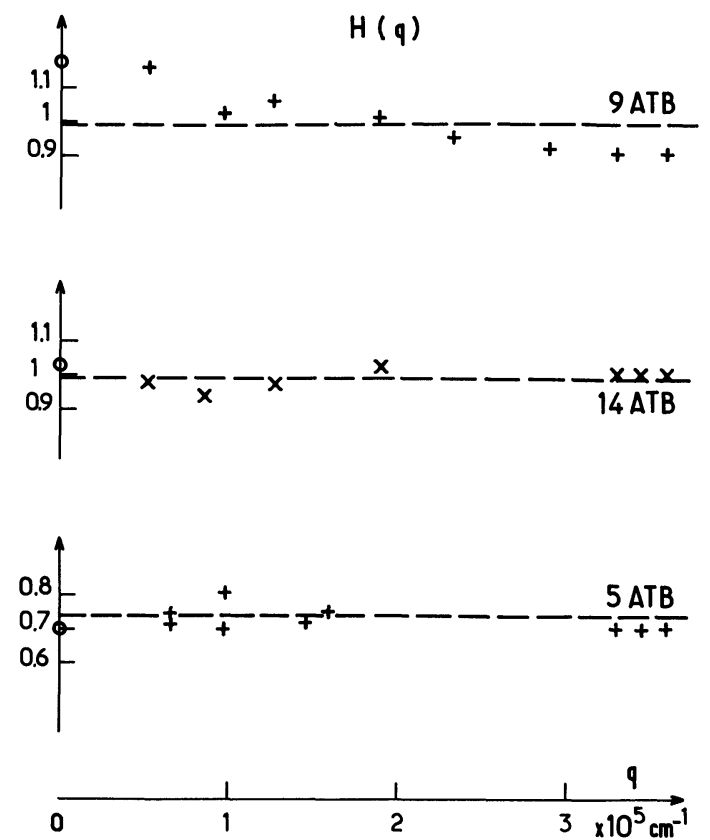

Fig. 6. $-q$ dependence of the hydrociyuaninis contribution $H$ for different volume fractions in microemulsions ATB.

and in the vicinity of a percolation threshold $\varphi_{\mathrm{c}}$ allowed us to obtain information abouit interaction ranges.

A structure factor variation was found : $S(q)$ decreases when $q$ increases. Similar observations have been made for other kinds of small interacting particles [11-13]. For instance in electrolyte solutions, $S(q)$ decreases when $q$ decreases. This has been interpreted in terms of long range repulsive interactions [11]. In microemulsions the variation of $S(q)$ indicates the existence of long range $(\sim 500 \AA)$ attractive interactions. Unfortunately, the nature of the interaction potential is not sufficiently well known to permit a comparison with experimental data. Far from the percolation threshold, it was pointed out that the nature of attractive interactions may arise from preferential soap chains solvatation effects $[1,6]$. However, such interactions are of much shorter range $\left(\sim R_{0}\right)$. It is clear that another interaction mechanism exists at least as one approaches the percolation threshold.

The increase of interaction range can probably be related to the divergence of the correlation length near the percolation threshold. However the interpretation of the data with percolation theory is not straightforward. A stirred percolation model should be developed for interacting spheres.

The problem of hydrodynamic interactions is even more complicated. But the experiments indicate that long range contributions are not important. The mechanism of hydrodynamic interactions can therefore be expected not to be affected by percolation.

The study of the influence of interaction strength on droplet interaction range is currently under way to try to elucidate these problems. 


\section{References}

[1] Calje, A. A., Atgerof, W. G. M., VriJ, A., in Micellization, Solubilization and Microemulsions, Vol. 2 (Plenum Press) 1977.

[2] GraciaA, A. et al., J. Physique Lett. 38 (1977) L-253 and 39 (1978) L-235.

[3] Zulauf, M., Eicke, H. F., J. Phys. Chem. 83 (1979) 480.

[4] Candau, S., Boutiller, J., Candau, F., Polymer 20 (1979) 1237.

[5] Cazabat, A. M., Langevin, D., Pouchelon, A., J. Colloid Interface Sci. 73 (1980) 1.

[6] Cazabat, A. M., Langevin, D., Proceedings of the light scattering workshop, Milano (1979) (Plenum Press), to appear. Cazabat, A. M., Langevin, D., J. Chem. Phys., to appear.

[7] Belloce, A. M., et al., Opt. Acta, to appear.

[8] Candau, F. et al., Polymer 20 (1979) 1221.

[9] Lagües, M., OBer, R., TAuPIN, C., J. Physique Lett. 39 (1978) L-487.

LAGÜES, M., J. Physique Lett. 40 (1979) L-331.
[10] Berge, P., Calmettes, P., Laj, C., Volochine, B., Phys. Rev. Lett. 23 (1969) 693.

Swinney, H. L., Henry, D. L., Phys. Rev. A 8 (1973) 2586.

[11] Schaefer, D. W., Berne, B. J., Phys. Rev. Lett. 32 (1974) 1170.

[12] Brown, J. C. et al., J. Phys. A 8 (1975) 665.

Pusey, P. N., J. Phys. A 8 (1975) 1433.

[13] Fisnaut, H. M. et al., Chem. Phys. Lett. 59 (1978) 351.

[14] De Gennes, P. G., J. Physique Lett. 40 (1979) L-199.

[15] Coniglio, A., Stanley, H. E., Klein, W., Phys. Rev. Lett. 42 (1979) 518.

[16] Ackerson, B. J., J. Chem. Phys. 64 (1976) 242, 69 (1978) 684.

[17] Hess, W., KLeIN, R., Proceedings of the light scattering workshop, Milano (1979) (Plenum Press), to appear.

[18] Berne, B. J., Pecora, R., Dynamic Light Scattering (Wiley) 1976.

[19] KERKER, M., The scattering of light and other electromagnetic radiation (Ac. Press) 1969. 